\title{
A Fixed-Point Approach to the Hyers-Ulam Stability of Caputo-Fabrizio Fractional Differential Equations
}

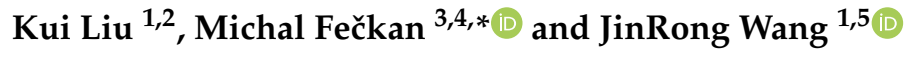 \\ 1 Department of Mathematics, Guizhou University, Guiyang 550025, China; \\ liuk180916@163.com (K.L.); jrwang@gzu.edu.cn (J.W.) \\ 2 College of Science, Guizhou Institute of Technology, Guiyang 550025, China \\ 3 Department of Mathematical Analysis and Numerical Mathematics, Faculty of Mathematics, Physics and \\ Informatics, Comenius University in Bratislava, Mlynská dolina, 84248 Bratislava, Slovakia \\ 4 Mathematical Institute, Slovak Academy of Sciences, Štefánikova 49, 81473 Bratislava, Slovakia \\ 5 School of Mathematical Sciences, Qufu Normal University, Qufu 273165, China \\ * Correspondence: michal.feckan@gmail.com or Michal.Feckan@fmph.uniba.sk
}

Received: 31 March 2020; Accepted: 19 April 2020; Published: 22 April 2020

\begin{abstract}
In this paper, we study Hyers-Ulam and Hyers-Ulam-Rassias stability of nonlinear Caputo-Fabrizio fractional differential equations on a noncompact interval. We extend the corresponding uniqueness and stability results on a compact interval. Two examples are given to illustrate our main results.
\end{abstract}

Keywords: Caputo-Fabrizio fractional differential equations; fixed-point theory; Hyers-Ulam stability

MSC: 26A33; 34D10; 45N05

\section{Introduction}

In 1940, Ulam posed a question concerning the stability of homomorphisms into metric groups, a question which is regarded as the origin of the problem of stability in the theory of functional equations. In 1941, Hyers [1] answered the problem for a linear functional equation on the Banach space and established a new concept on the stability of functional equation, now called Hyers-Ulam stability. In 1978, Rassias [2] introduced a new definition of generalized Hyers-Ulam stability by the constant $\varepsilon$ by a variable, and obtained the stability of Hyers-Ulam-Rassias for functional equation. There is a rich literature on this topic for standard integer-order equations (see [3-17]). In addition, the same stability concepts are introduced to find approximate solutions to fractional differential equations, see [18,19] and the references therein.

In 2015, Caputo and Fabrizio [20] gave a new definition of fractional derivative with a smooth kernel. Losada and Nieto [21] introduced Caputo-Fabrizio fractional differential equation the newly developed Caputo-Fabrizio fractional derivative and obtained the existence and uniqueness results under some strong restriction. Baleanu et al. [22] obtained the approximate solution for some infinite coefficient-symmetric Caputo-Fabrizio fractional integro-differential equations. Goufo [23] used the fractional derivative of the newly developed Caputo-Fabrizio without singular kernel to establish the Korteweg-de Vries-Burgers equation with two perturbation levels. Atangana and Nieto [24] studied the numerical approximation of this new fractional derivative and established an improved RLC circuit model. Moore et al. [25] developed and analyzed a Caputo-Fabrizio fractional derivative model for the HIV epidemic which includes an antiretroviral treatment compartment. Dokuyucu et al. [26] applied the fractional derivative of Caputo-Fabrizio to model the cancer treatment by radiotherapy. 
Recently, Başc1 et al. [27] applied the Laplace transform method to study the Hyers-Ulam stability of the following linear differential equations with Caputo-Fabrizio fractional derivative (see Definition 1):

$$
\left({ }^{C F} \mathbb{D}^{\alpha} y\right)(t)=f(t), 0<\alpha<1,
$$

and

$$
\left({ }^{C F} \mathbb{D}^{\alpha} y\right)(t)-\lambda y(t)=f(t), 0<\alpha<1 .
$$

Meanwhile, Liu et al. [4] presented the Hyers-Ulam stability of linear differential equations with two term Caputo-Fabrizio derivatives as follows

$$
\left({ }^{C F} \mathbb{D}^{\alpha} y\right)(t)-\lambda\left({ }^{C F} \mathbb{D}^{\beta} y\right)(t)=u(t), 0<\alpha, \beta<1,
$$

and applied fixed-point theorems to derive the existence and uniqueness of solution to nonlinear equations as follows

$$
\left({ }^{C F} \mathbb{D}^{\alpha} f\right)(t)=g(t, f(t)), 0<\alpha<1,
$$

and obtained the generalized Hyers-Ulam-Rassias stability via the Gronwall's inequality.

Observing that ([4], Theorem 3) adopted the generalized Banach fixed-point theorem instead of the standard Banach contraction mapping and weakened the condition $a_{\alpha} L+b_{\alpha} T L<1$ in ([21], Theorem 1) to $a_{\alpha} L<1$ where $k>0$ denoted by the Lipschtiz constant of $g, T$ denoted by the step of the interval and

$$
a .=\frac{2(1-\cdot)}{(2-\cdot) M(\cdot)}, \quad b .=\frac{2 \cdot}{(2-\cdot) M(\cdot)}
$$

and $M(\cdot)$ denotes a normalization constant depending on $\cdot$

Based on the above observation, we apply a new fixed-point approach to show the existence and uniqueness and stability for (1) on a compact interval to a noncompact interval $J=\left[\tau_{0}, \tau_{0}+k\right), k>0$.

\section{Preliminaries}

Definition 1 (see [20]). Let $0<\gamma<1$, the Caputo-Fabrizio fractional derivative of order $\gamma$ for a function $f$ can be written as

$$
{ }^{C F} \mathbb{D}^{\gamma} f(\tau)=\frac{(2-\gamma) M(\gamma)}{2(1-\gamma)} \int_{a}^{\tau} \exp \left(-\frac{\gamma}{1-\gamma}(\tau-s)\right) f^{\prime}(s) d s, \tau>a,
$$

where $M(\gamma)$ is a normalization constant depending on $\gamma$. Please note that $\left({ }^{C F} \mathbb{D}^{\gamma}\right)(f)=0$ if and only if $f$ is a constant function.

Definition 2 (see [21] or ([4], Definition 2)). Let $0<\gamma<1$. The Caputo-Fabrizio fractional integral of order $\gamma$ for a function $f$ is defined as

$$
{ }^{C F} I^{\gamma} f(\tau)=\frac{2(1-\gamma)}{(2-\gamma) M(\gamma)} f(\tau)+\frac{2 \gamma}{(2-\gamma) M(\gamma)} \int_{a}^{\tau} f(s) d s, \tau>a .
$$

Let $\Omega$ be a nonempty set, we present the following definition of generalized metric on $\Omega$.

Definition 3 (see [3]). A function $\rho: \Omega \times \Omega \rightarrow[0, \infty]$ is called a generalized metric on $\Omega$ if and only if $\rho$ satisfies 
(i) $\rho\left(\tau_{1}, \tau_{2}\right)=0$ if and only if $\tau_{1}=\tau_{2}$;

(ii) $\rho\left(\tau_{1}, \tau_{2}\right)=\rho\left(\tau_{2}, \tau_{1}\right)$ for all $\tau_{1}, \tau_{2} \in \Omega$;

(iii) $\rho\left(\tau_{1}, \tau_{3}\right) \leq \rho\left(\tau_{1}, \tau_{2}\right)+\rho\left(\tau_{2}, \tau_{3}\right)$ for all $\tau_{1}, \tau_{2}, \tau_{3} \in \Omega$;

Theorem 1 (see [28]). Let $(\Omega, \rho)$ is a generalized complete metric space. Suppose $P: \Omega \rightarrow \Omega$ is a strictly contractive operator with the Lipschitz constant $K<1$. If there exists a nonnegative integer $l$ such that $\rho\left(P^{l+1} \tau, P^{l} \tau\right)<\infty$ for some $\tau \in \Omega$, then the followings are true:

(i) The sequence $\left\{P^{n} \tau\right\}$ converges to a fixed point $\tau^{*}$ of $P$;

(ii) $\tau^{*}$ is the unique fixed point of $P$ in

$$
\Omega^{*}=\left\{\tilde{\tau} \in \Omega \mid \rho\left(P^{l} \tau, \tilde{\tau}\right)<\infty\right\} ;
$$

(iii) If $\tilde{\tau} \in \Omega^{*}$, then

$$
\rho\left(\tilde{\tau}, \tau^{*}\right) \leq \frac{1}{1-K} \rho(P \tilde{\tau}, \tilde{\tau}) .
$$

Definition 4 (see [4]). Let $g: J \times \mathbb{R} \rightarrow \mathbb{R}$ be a continuous function. Equation (7) is Hyers-Ulam stable if there exists a real number $N>0$, such that for each $\epsilon>0$ and for any solution $f \in C(J, \mathbb{R})$ of

$$
\left|{ }^{C F} \mathbb{D}^{\gamma} f(\tau)-g(\tau, f(\tau))\right| \leq \epsilon, \forall \tau \in J,
$$

there exists a solution $h \in C(J, \mathbb{R})$ of (1) with

$$
|f(\tau)-h(\tau)| \leq N \epsilon, \forall \tau \in J .
$$

Definition 5 (see [4]). Let $\phi: J \rightarrow \mathbb{R}_{+}$and $g: J \times \mathbb{R} \rightarrow \mathbb{R}$ be continuous functions. Equation (7) is generalized Hyers-Ulam-Rassias stable with respect to $\phi \in C\left(J, \mathbb{R}_{+}\right)$, if there exists a constant $c_{f, \phi}>0$ such that for any solution $f \in C(J, \mathbb{R})$ of

$$
\left|{ }^{C F} \mathbb{D}^{\gamma} f(\tau)-g(\tau, f(\tau))\right| \leq \phi(\tau), \forall \tau \in J,
$$

there exists a solution $h \in C(J, \mathbb{R})$ of (1) with

$$
|f(\tau)-h(\tau)| \leq c_{f, \phi} \phi(\tau), \forall \tau \in J
$$

\section{Main Results}

Throughout this section, we denote the set $Y$ of all continuous functions on $J$ by

$$
Y:=\{g: J \rightarrow \mathbb{R} \mid g \text { is continuous }\}=C(J, \mathbb{R})
$$

Lemma 1 (see ([3], Theorem 3.1)). Define the function $d: Y \times Y \rightarrow[0, \infty]$ with

$$
d(f, g):=\inf \{M \in[0, \infty]|| f(\tau)-g(\tau) \mid \leq M \psi(\tau), \forall \tau \in J\}
$$

where $\psi: J \rightarrow[0, \infty)$ is a given continuous function. Then $(Y, d)$ is a generalized complete metric space.

We give the following conditions:

$\left[A_{1}\right]$ The function $g: J \times \mathbb{R} \rightarrow \mathbb{R}$ is continuous and locally Lipschitz in $\tau$.

$\left[A_{2}\right]$ There exists a constant $L>0$ such that

$$
\left|g\left(\tau, y_{1}\right)-g\left(\tau, y_{2}\right)\right| \leq L\left|y_{1}-y_{2}\right|, \quad \forall y_{1}, y_{2} \in \mathbb{R}, \tau \in J .
$$


Now, we prove the Hyers-Ulam stability of (7).

Theorem 2. Assume that $\left[A_{1}\right]$ and $\left[A_{2}\right]$ and $\left|a_{\gamma}\right|<1 /(L+1)$ hold. If the function $h: J \rightarrow \mathbb{R}$ is continuously differentiable and satisfies

$$
\left|\left({ }^{C F} \mathbb{D}^{\gamma} h\right)(\tau)-g(\tau, h(\tau))\right| \leq \epsilon
$$

for all $\tau \in J$ and for some $\epsilon>0$, then there exists a unique solution $f(\tau)$ of

$$
\left({ }^{C F} \mathbb{D}^{\gamma} f\right)(\tau)=g(\tau, f(\tau)), 0<\gamma<1,
$$

satisfying

$$
|h(\tau)-f(\tau)| \leq(L+1)\left(\left|a_{\gamma}\right|+\left|b_{\gamma}\right| k\right) \epsilon
$$

for all $\tau \in J$, where $a_{\gamma}$ and $b_{\gamma}$ are defined in (2).

Proof. We introduce a function $d_{1}: Y \times Y \rightarrow[0, \infty]$, where $Y$ defined by (5) with

$$
d_{1}(f, g):=\inf \left\{M \in[0, \infty]|| f(\tau)-g(\tau) \mid e^{-K\left(\tau-\tau_{0}\right)} \leq M, \forall \tau \in J\right\},
$$

where $K=\frac{(L+1)\left|b_{\gamma}\right|}{1-(L+1)\left|a_{\gamma}\right|}>0$ and $a_{\gamma}, b_{\gamma}$ are given in (2)

Let $\psi(\cdot)=e^{K\left(\cdot-\tau_{0}\right)}$ in Lemma 1 , we obtain $\left(Y, d_{1}\right)$ is a generalized complete metric space.

Next, we consider the operator $P: Y \rightarrow Y$ as follows:

$$
(P f)(\tau):=f_{0}+a_{\gamma} g(\tau, f(\tau))+b_{\gamma} \int_{\tau_{0}}^{\tau} g(s, f(s)) d s, \quad \tau \in J .
$$

for any $f, g \in Y$, where $f_{0}=f\left(\tau_{0}\right)$. Please note that any fixed point of $P$ solves (7). Indeed, the function $u-a_{\gamma} g(\tau, u)=v$ in (10) is invertible, it is increasing. We denote its inverse $u=G(\tau, v)$, and $G$ is globally Lipschitz in $v$ and locally Lipschitz in $\tau$ by our assumptions. So, any fixed point of (10) satisfies

$$
f(\tau)=G\left(\tau, b_{\gamma} \int_{\tau_{0}}^{\tau} g(s, f(s)) d s+f_{0}\right) .
$$

Now clearly the function $\tau \rightarrow b_{\gamma} \int_{\tau_{0}}^{\tau} g(s, f(s)) d s+f_{0}$ is locally Lipschitz in $\tau$, we see that the composition function $\tau \rightarrow G\left(\tau, b_{\gamma} \int_{\tau_{0}}^{\tau} g(s, f(s)) d s+f_{0}\right)$ is also locally Lipschitz in $\tau$. So, any fixed point $f(\tau)$ of $(10)$ is a locally Lipschitz function, and thus it is locally absolute continuous on $J$. So really (10) gives solutions of (7). As a matter of fact, we need just that $u-a_{\gamma} g(\tau, u)=v$ is invertible, i.e., $u-a_{\gamma} g(\tau, u)$ is strictly monotonic in $u$, and we can extend our results for more general case. We shall consider (11) instead of (10). 
We prove that $P f$ is continuous. Let $\tau_{1}, \tau_{2} \in J$, and $\tau_{1}<\tau_{2}$, we have

$$
\begin{aligned}
& \left|P f\left(\tau_{1}\right)-P f\left(\tau_{2}\right)\right| \\
= & \left|a_{\gamma} g\left(\tau_{1}, f\left(\tau_{1}\right)\right)+b_{\gamma} \int_{\tau_{0}}^{\tau_{1}} g(s, f(s)) d s-a_{\gamma} g\left(\tau_{2}, f\left(\tau_{2}\right)\right)-b_{\gamma} \int_{\tau_{0}}^{\tau_{2}} g(s, f(s)) d s\right| \\
\leq & \left|a_{\gamma}\right|\left|g\left(\tau_{1}, f\left(\tau_{1}\right)\right)-g\left(\tau_{2}, f\left(\tau_{2}\right)\right)\right|+\left|b_{\gamma}\right|\left|\int_{\tau_{0}}^{\tau_{1}} g(s, f(s)) d s-\int_{\tau_{0}}^{\tau_{2}} g(s, f(s)) d s\right| \\
\leq & \left|a_{\gamma}\right|\left|g\left(\tau_{1}, f\left(\tau_{1}\right)\right)-g\left(\tau_{1}, f\left(\tau_{2}\right)\right)\right|+\left|a_{\gamma}\right|\left|g\left(\tau_{1}, f\left(\tau_{2}\right)\right)-g\left(\tau_{2}, f\left(\tau_{2}\right)\right)\right|+\left|b_{\gamma}\right|\left|\int_{\tau_{1}}^{\tau_{2}} g(s, f(s)) d s\right| \\
\leq & \left|a_{\gamma}\right|\left|g\left(\tau_{1}, f\left(\tau_{1}\right)\right)-g\left(\tau_{1}, f\left(\tau_{2}\right)\right)\right|+\left|a_{\gamma}\right|\left|g\left(\tau_{1}, f\left(\tau_{2}\right)\right)-g\left(\tau_{2}, f\left(\tau_{2}\right)\right)\right| \\
& +\left|b_{\gamma}\right|\left(\int_{\tau_{1}}^{\tau_{2}}|g(s, f(s))-g(s, 0)| d s+\int_{\tau_{1}}^{\tau_{2}}|g(s, 0)| d s\right) \\
\leq & \left|a_{\gamma}\right|\left|g\left(\tau_{1}, f\left(\tau_{1}\right)\right)-g\left(\tau_{1}, f\left(\tau_{2}\right)\right)\right|+\left|a_{\gamma}\right|\left|g\left(\tau_{1}, f\left(\tau_{2}\right)\right)-g\left(\tau_{2}, f\left(\tau_{2}\right)\right)\right| \\
& +\left|b_{\gamma}\right|\left(L\|f\|_{C(J, \mathbb{R})}\left(\tau_{2}-\tau_{1}\right)+\|g\| C(J, \mathbb{R})\left(\tau_{2}-\tau_{1}\right)\right) .
\end{aligned}
$$

Then, for all $f \in Y$, as $\tau_{1} \rightarrow \tau_{2}$, the right-hand side of the above inequality tends to zero (due to $\left[A_{1}\right]$ and $\left.f \in Y\right)$. Thus, $P f$ is continuous, i.e., $P f \in Y$ for all $f \in Y$.

Then, we have

$$
\left|\left(P f_{0}\right)(\tau)-f_{0}(\tau)\right| e^{-K\left(\tau-\tau_{0}\right)} \leq\left\|P f_{0}-f_{0}\right\|_{C(J, \mathbb{R})} \max \left\{1, e^{-K k}\right\}<\infty,
$$

for all $f_{0} \in Y$, and $\tau \in J$. Therefore, by (9), we obtain $d_{1}\left(P f_{0}, f_{0}\right)<\infty, f_{0} \in Y$.

Similarly, we have

$$
\left|\left(f_{0}\right)(\tau)-f(\tau)\right| e^{-K\left(\tau-\tau_{0}\right)} \leq\left\|f_{0}-f\right\|_{C(J, \mathbb{R})} \max \left\{1, e^{-K k}\right\}<\infty,
$$

for all $f \in Y$, and $\tau \in J$, which implies that

$$
d_{1}\left(f_{0}, f\right)<\infty, \forall f \in Y,
$$

that is $\left\{f \in Y \mid d_{1}\left(f_{0}, f\right)<\infty\right\}=Y$.

Next, we show that $P$ is strictly contractive on $Y$. For any $l, n \in Y$, we get

$$
\begin{aligned}
& |(P l)(\tau)-(P n)(\tau)| \\
\leq & \left|a_{\gamma}\right||g(\tau, l(\tau))-g(\tau, n(\tau))|+\left|b_{\gamma}\right| \int_{\tau_{0}}^{\tau}|g(s, l(s))-g(s, n(s))| d s \\
\leq & L\left|a_{\gamma}\right||l(\tau)-n(\tau)|+L\left|b_{\gamma}\right| \int_{\tau_{0}}^{\tau}|l(s)-n(s)| d s \\
\leq & L\left|a_{\gamma}\right||l(\tau)-n(\tau)|+L\left|b_{\gamma}\right| \int_{\tau_{0}}^{\tau}|l(s)-n(s)| e^{-K\left(s-\tau_{0}\right)} e^{K\left(s-\tau_{0}\right)} d s \\
\leq & L\left|a_{\gamma}\right||l(\tau)-n(\tau)|+L\left|b_{\gamma}\right| d_{1}(l, n) \int_{\tau_{0}}^{\tau} e^{K\left(s-\tau_{0}\right)} d s \\
\leq & L\left|a_{\gamma}\right||l(\tau)-n(\tau)|+\frac{L\left|b_{\gamma}\right|}{K} d_{1}(l, n)\left(e^{K\left(\tau-\tau_{0}\right)}-1\right) \\
\leq & L\left|a_{\gamma}\right||l(\tau)-n(\tau)|+\frac{L\left|b_{\gamma}\right|}{K} d_{1}(l, n) e^{K\left(\tau-\tau_{0}\right)}
\end{aligned}
$$


for all $\tau \in J$. Thus, for any $l, n \in Y$ and all $\tau \in J$, we have

$$
\begin{aligned}
|(P l)(\tau)-(P n)(\tau)| e^{-K\left(\tau-\tau_{0}\right)} & \leq L\left|a_{\gamma}\right||l(\tau)-n(\tau)| e^{-K\left(\tau-\tau_{0}\right)}+\frac{L\left|b_{\gamma}\right|}{K} d_{1}(l, n) \\
& \leq L\left|a_{\gamma}\right| d_{1}(l, n)+\frac{L\left|b_{\gamma}\right|}{K} d_{1}(l, n) \\
& =L\left(\left|a_{\gamma}\right|+\frac{\left|b_{\gamma}\right|}{K}\right) d_{1}(l, n) \\
& =\frac{L}{L+1} d_{1}(l, n) .
\end{aligned}
$$

Hence, we obtain

$$
d_{1}(P l, P n) \leq \frac{L}{L+1} d_{1}(l, n) .
$$

Therefore, $P$ is strictly contractive on $Y$.

When $k=1$ and $Y=\Omega^{*}$, the operator $P$ satisfies all the conditions of Theorem 1 .

On the other hand, by (6), we have

$$
-\epsilon \leq\left({ }^{C F} \mathbb{D}^{\gamma} h\right)(\tau)-g(\tau, h(\tau)) \leq \epsilon \forall \tau \in J .
$$

Similar to the approach in ([4], Theorem 2), we can obtain

$$
\left|h(\tau)-h_{0}-a_{\gamma} g(\tau, h(\tau))-b_{\gamma} \int_{\tau_{0}}^{\tau} g(s, f(s)) d s\right| \leq \epsilon\left(\left|a_{\gamma}\right|+\left|b_{\gamma}\right| k\right)
$$

for all $\tau \in J$. From (10), (12) is equivalent to

$$
|h(\tau)-(P h)(\tau)| \leq \epsilon\left(\left|a_{\gamma}\right|+\left|b_{\gamma}\right| k\right) .
$$

Multiply both sides of (13) by $e^{-K\left(\tau-\tau_{0}\right)}$,

$$
|h(\tau)-(P h)(\tau)| e^{-K\left(\tau-\tau_{0}\right)} \leq \epsilon\left(\left|a_{\gamma}\right|+\left|b_{\gamma}\right| k\right) e^{-K\left(\tau-\tau_{0}\right)} \leq M:=\epsilon\left(\left|a_{\gamma}\right|+\left|b_{\gamma}\right| k\right) \max \left\{1, e^{-K k}\right\}
$$

for all $\tau \in J$. Then

$$
d_{1}(P h, h) \leq \epsilon\left(\left|a_{\gamma}\right|+\left|b_{\gamma}\right| k\right) e^{-K\left(\tau-\tau_{0}\right)} .
$$

By Theorem 1, there exists a unique solution $f: J \rightarrow \mathbb{R}$ of (7) satisfying

$$
d_{1}(h, f) \leq \frac{1}{1-L /(L+1)} d_{1}(P h, h) \leq(L+1) \epsilon\left(\left|a_{\gamma}\right|+\left|b_{\gamma}\right| k\right) e^{-K\left(\tau-\tau_{0}\right)}, \quad \tau \in J,
$$

by (9), we have

$$
|h(\tau)-f(\tau)| e^{-K\left(\tau-\tau_{0}\right)} \leq(L+1) \epsilon\left(\left|a_{\gamma}\right|+\left|b_{\gamma}\right| k\right) e^{-K\left(\tau-\tau_{0}\right)}, \quad \tau \in J,
$$

which implies that (8) holds.

Remark 1. From Definition 4, (8) shows (7) is Hyers-Ulam stable with the constant $N=(L+1)\left(\left|a_{\gamma}\right|+\left|b_{\gamma}\right| k\right)$ provided that $0<k<+\infty$. Of course, (7) is not Hyers-Ulam stable if $k=+\infty$. Theorem 2 covers the result in ([27], Theorem 2.6) and shows that the condition $0<\lambda<\frac{(2-\alpha) M(\alpha)}{2(1-\alpha)}$ can be removed.

Now we will prove the Hyers-Ulam-Rassias stability of (7). 
Theorem 3. Assume that $\left[A_{1}\right]$ and $\left[A_{2}\right]$ and $\left|a_{\gamma}\right|<1 /(L+1)$ hold. If a continuously differentiable function $h: J \rightarrow \mathbb{R}$ satisfies

$$
\left|\left({ }^{C F} \mathbb{D}^{\gamma} h\right)(\tau)-g(\tau, h(\tau))\right| \leq G(\tau)
$$

for all $\tau \in J$ and for some $G: J \rightarrow(0, \infty)$ is a nondecreasing continuous function satisfying

$$
\left|\int_{\tau_{0}}^{\tau} G(s) d s\right| \leq F_{G} G(\tau), F_{G}>0,
$$

for all $\tau \in J$, then there exists a unique solution $f(\tau)$ of (7) satisfying

$$
|h(\tau)-f(\tau)| \leq(L+1)\left(a_{\gamma}+b_{\gamma} F_{G}\right) G(\tau)
$$

for all $\tau \in J$.

Proof. We introduce a function $d_{2}: Y \times Y \rightarrow[0, \infty]$, where $Y$ defined by (5) with

$$
d_{2}(f, g):=\inf \left\{M \in[0, \infty]|| f(\tau)-g(\tau) \mid e^{-K\left(\tau-\tau_{0}\right)} \leq M G(\tau), \forall \tau \in J, K \in \mathbb{R}\right\}
$$

Let $\psi(\cdot)=e^{K\left(\cdot-\tau_{0}\right)} G(\cdot)$ in the Lemma $1,\left(Y, d_{2}\right)$ is a generalized complete metric space.

Consider $P: Y \rightarrow Y$ defined in (10). Similar to the method of Theorem 2, we can conclude that $d_{2}\left(P f_{0}, f\right)<\infty$ for each $f_{0} \in X$ and $\left\{f \in Y \mid d_{2}\left(f_{0}, f\right)<\infty\right\}=Y$.

Next, we prove that $P$ is strictly contractive on $Y$. Note

$$
\begin{aligned}
\int_{\tau_{0}}^{\tau} G(s) e^{K\left(s-\tau_{0}\right)} d s & \leq G(\tau) \int_{\tau_{0}}^{\tau} e^{K\left(s-\tau_{0}\right)} d s \\
& =\frac{1}{K} G(\tau) \int_{\tau_{0}}^{\tau} d e^{K\left(s-\tau_{0}\right)} \\
& \leq \frac{1}{K} G(\tau)\left(e^{K\left(\tau-\tau_{0}\right)}-1\right) \\
& \leq \frac{1}{K} G(\tau) e^{K\left(\tau-\tau_{0}\right)}
\end{aligned}
$$

for all $\tau \in J$.

For any $l, n \in Y$, let $M_{l, n} \in[0, \infty]$ be an arbitrary constant with $d_{2}(l, n) \leq M_{l, n}$, by (17), we obtain

$$
|l(\tau)-n(\tau)| e^{-K\left(\tau-\tau_{0}\right)} \leq M_{l, n} G(\tau), \text { for all } \tau \in J .
$$

Then, for each $l, n \in Y$, we have

$$
\begin{aligned}
& |(P l)(\tau)-(P n)(\tau)| \\
\leq & \left|a_{\gamma}\right||g(\tau, l(\tau))-g(\tau, n(\tau))|+\left|b_{\alpha}\right| \int_{\tau_{0}}^{\tau}|g(s, l(s))-g(s, n(s))| d s \\
\leq & L\left|a_{\gamma}\right||l(\tau)-n(\tau)|+L\left|b_{\gamma}\right| \int_{\tau_{0}}^{\tau}|l(s)-n(s)| d s \\
\leq & L\left|a_{\gamma}\right||l(\tau)-n(\tau)|+L\left|b_{\gamma}\right| \int_{\tau_{0}}^{\tau}|l(s)-n(s)| e^{-K\left(s-\tau_{0}\right)} e^{K\left(s-\tau_{0}\right)} d s \\
\leq & L\left|a_{\gamma}\right||l(\tau)-n(\tau)|+L\left|b_{\gamma}\right| M_{l, n} \int_{\tau_{0}}^{\tau} G(s) e^{K\left(s-\tau_{0}\right)} d s \\
\leq & L\left|a_{\gamma}\right||l(\tau)-n(\tau)|+L\left|b_{\gamma}\right| M_{l, n} \frac{1}{K} G(\tau) e^{K\left(\tau-\tau_{0}\right)}
\end{aligned}
$$


for all $\tau \in J$. Thus, for any $l, n \in Y$ and all $\tau \in J$, we have

$$
\begin{aligned}
|(P l)(\tau)-(P n)(\tau)| e^{-K\left(\tau-\tau_{0}\right)} & \leq L\left|a_{\gamma}\right||l(\tau)-n(\tau)| e^{-K\left(\tau-\tau_{0}\right)}+\frac{L\left|b_{\gamma}\right|}{K} M_{l, n} G(\tau) \\
& \leq L\left|a_{\gamma}\right| M_{l, n} G(\tau)+\frac{L\left|b_{\gamma}\right|}{K} M_{l, n} G(\tau) \\
& =L\left(\left|a_{\gamma}\right|+\frac{\left|b_{\gamma}\right|}{K}\right) M_{l, n} G(\gamma) \\
& =\frac{L}{L+1} M_{l, n} G(\tau),
\end{aligned}
$$

that is, $d_{2}(P l, P n) \leq \frac{L}{L+1} M_{l, n}, \forall \tau \in J$. Hence, we obtain

$$
d_{2}(P l, P n) \leq \frac{L}{L+1} d_{2}(l, n), \quad \forall \tau \in J .
$$

Therefore, $P$ is strictly contractive on $Y$. When $k=1$ and $Y=\Omega^{*}$, the operator $P$ satisfies all the conditions of Theorem 1 .

On the other hand, by (14), we have

$$
-G(\tau) \leq\left({ }^{C F} \mathbb{D}^{\gamma} h\right)(\tau)-g(\tau, h(\tau)) \leq G(\tau), \quad \forall \tau \in J .
$$

By simple computation, we can obtain

$$
\begin{aligned}
& \left|h(\tau)-h_{0}-a_{\gamma} g(\tau, h(\tau))-b_{\gamma} \int_{\tau_{0}}^{\tau} g(s, f(s)) d s\right| \\
\leq & \left|a_{\gamma}\right| G(\tau)+\left|b_{\gamma}\right| \int_{\tau_{0}}^{\tau} G(s) d s \\
\leq & \left(\left|a_{\gamma}\right|+\left|b_{\gamma}\right| F_{G}\right) G(\tau), \forall \tau \in J .
\end{aligned}
$$

This yields that

$$
|h(\tau)-(P h)(\tau)| \leq\left(\left|a_{\gamma}\right|+\left|b_{\gamma}\right| F_{G}\right) G(\tau), \quad \forall \tau \in J .
$$

Multiply both sides of (18) by $e^{-K\left(\tau-\tau_{0}\right)}$, then,

$$
|h(\tau)-(P h)(\tau)| e^{-K\left(\tau-\tau_{0}\right)} \leq\left(\left|a_{\gamma}\right|+\left|b_{\gamma}\right| F_{G}\right) G(\tau) e^{-K\left(\tau-\tau_{0}\right)}, \quad \forall \tau \in J .
$$

Then

$$
d_{2}(P h, h) \leq\left(\left|a_{\gamma}\right|+\left|b_{\gamma}\right| F_{G}\right) G(\tau) e^{-K\left(\tau-\tau_{0}\right)}, \quad \forall \tau \in J .
$$

By Theorem 1 , there exists a unique solution $f: J \rightarrow \mathbb{R}$ of (7) satisfying

$$
d_{2}(h, f) \leq \frac{1}{1-L /(L+1)} d_{2}(P h, h) \leq(L+1)\left(\left|a_{\gamma}\right|+\left|b_{\gamma}\right| F_{G}\right) G(\tau) e^{-K\left(\tau-\tau_{0}\right)}, \quad \forall \tau \in J .
$$

By (17), we have

$$
|h(\tau)-f(\tau)| e^{-K\left(\tau-\tau_{0}\right)} \leq(L+1)\left(\left|a_{\gamma}\right|+\left|b_{\gamma}\right| F_{G}\right) G(\tau) e^{-K\left(\tau-\tau_{0}\right)}, \quad \forall \tau \in J,
$$

which implies (16) holds. The proof is complete.

Remark 2. By the Definition 5, (16) shows (7) is generalized Hyers-Ulam-Rassias stable with the constant $c_{f, G}=(L+1)\left(\left|a_{\gamma}\right|+\left|b_{\gamma}\right| F_{G}\right)$. Theorem 3 extend the result in ([27], Corollary 2.8) and also shows that the condition $0<\lambda<\frac{(2-\alpha) M(\alpha)}{2(1-\alpha)}$ can be removed. 
Remark 3. Compared to ([4], Theorems 3 and 5), we extend the existence and uniqueness result and the generalized Hyers-Ulam-Rassias stability result for (1) on the noncompact interval and also remove the condition $L\left|a_{\alpha}\right|<1$ from the assumptions.

\section{Examples}

Assume that $M(\cdot)$ in Definition 1 is the solution of the following equation:

$$
\frac{2(1-\cdot)}{(2-\cdot) M(\cdot)}+\frac{2 \cdot}{(2-\cdot) M(\cdot)}=1
$$

Then one can derive an explicit formula $M(\cdot)=\frac{2}{2-\text {. }}($ see $([21]$, p. 89)).

Example 1. We consider the following equation:

$$
\left({ }^{C F} \mathbb{D}^{\gamma} f\right)(\tau)-\lambda f(\tau)=g(\tau), \tau \in[0, k), k>0,
$$

and let $g(\tau, f(\tau))=g(\tau)+\lambda f(\tau)$. Obviously, $\left|g\left(\tau, f_{1}(\tau)\right)-g\left(\tau, f_{2}(\tau)\right)\right|=|\lambda|\left|f_{1}(\tau)-f_{2}(\tau)\right|, \tau \in[0, k)$ and the Lipschitz condition holds with the Lipschitz constant $L=|\lambda|$. Then, (19) is Hyers-Ulam stable on $J$, for all $\lambda \in \mathbb{R}$ and $\alpha \in(0,1)$.

Now, let $\gamma=\frac{1}{2}, \lambda=-2, f(0)=0$, and $g(\tau)=4 \tau-4+4 e^{-\tau}-\frac{1}{2} e^{-2 \tau}+2 \tau^{2}$. We consider the following equation:

$$
\left({ }^{C F} \mathbb{D}^{\frac{1}{2}} f\right)(\tau)+2 f(\tau)=g(\tau), \tau \in[0, k), k>0 .
$$

Let $h(\tau)=\tau^{2}$, for $\epsilon=\frac{1}{2}$ by simple calculation, we have

$$
\left({ }^{C F} \mathbb{D}^{\frac{1}{2}} h\right)(\tau)=4 \tau-4+4 e^{-\tau}
$$

then

$$
\left|\left({ }^{C F} \mathbb{D}^{\frac{1}{2}} h\right)(\tau)+2 f(\tau)-g(\tau)\right|=\frac{1}{2} e^{-2 \tau} \leq \frac{1}{2}=\epsilon, \tau \in[0, k), k>0 .
$$

Integrating (20) from 0 to $\tau$, we get

$$
f(\tau)=\tau^{2}-\frac{1}{12} e^{-2 \tau}+\frac{1}{12} e^{-\frac{1}{2} \tau}
$$

then

$$
\begin{aligned}
|h(\tau)-f(\tau)| & =\left|\frac{1}{12} e^{-2 \tau}-\frac{1}{12} e^{-\frac{1}{2} \tau}\right|=\frac{1}{12} e^{-\frac{1}{2} \tau}\left|1-e^{-\frac{3}{2} \tau}\right| \\
& \leq \frac{1}{6} \times \frac{1}{2}=\frac{1}{6} \epsilon .
\end{aligned}
$$

So (20) is Hyers-Ulam stable (see Figure 1). Please note that the condition $\lambda>0$ in ([27], Theorem 2.6) is not required here, and moreover, (20) is Hyers-Ulam stable, too.

On the other hand, (21) implies that (20) is also Hyers-Ulam stable even for $\tau=+\infty$, which shows that ([27], Remark 2.7) is not suitable. 


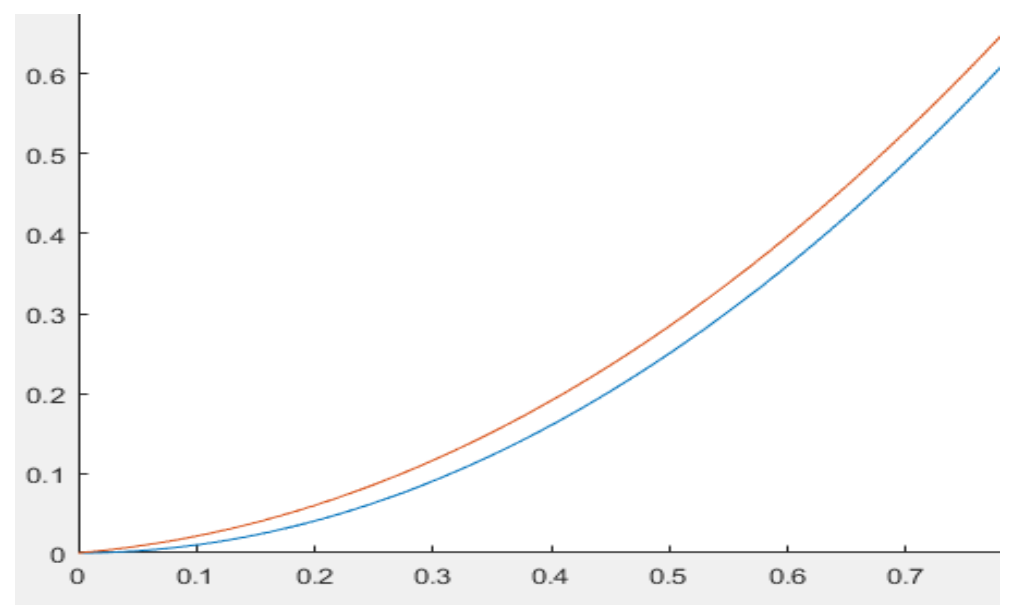

Figure 1. The exact and approximated solutions of the differential equation (20) are shown by the red and blue lines, respectively.

Example 2. We consider the following fractional problem

$$
\left({ }^{C F} \mathbb{D}^{\frac{1}{3}} f\right)(\tau)=\frac{5}{1+e^{\tau}} \frac{|f|}{1+|f|}, \tau \in[0,+\infty),
$$

and the inequality

$$
\left|\left({ }^{C F} \mathbb{D}^{\frac{1}{3}} f\right)(\tau)-\frac{5}{1+e^{\tau}} \frac{|f|}{1+|f|}\right| \leq G(\tau), \tau \in[0,+\infty) .
$$

Let $g(\tau, f(\tau))=\frac{5}{1+e^{\tau}} \frac{|f|}{1+|f|},(\tau, f) \in[0,+\infty) \times \mathbb{R}$. Obviously $\left[A_{1}\right]$ holds. For any $\tau \in[0,+\infty)$ and $f_{1}, f_{2} \in \mathbb{R}$, we have

$$
\begin{aligned}
\left|g\left(\tau, f_{1}\right)-g\left(\tau, f_{2}\right)\right| & =\frac{5}{1+e^{\tau}}\left|\frac{\left|f_{1}\right|}{1+\left|f_{1}\right|}-\frac{\left|f_{2}\right|}{1+\left|f_{2}\right|}\right| \leq \frac{5\left|f_{1}-f_{2}\right|}{\left(1+\left|f_{1}\right|\right)\left(1+\left|f_{2}\right|\right)} \\
& \leq 5\left|f_{1}-f_{2}\right| .
\end{aligned}
$$

Then the condition $\left[A_{2}\right]$ hold and $L=5$ and $k_{\alpha}=5$ in ([4], Theorem 5).

Let $G(\tau)=e^{\tau} \in C([0,+\infty),(0,+\infty))$ and $\int_{0}^{\tau} G(s) d s=\int_{0}^{\tau} e^{s} d s=e^{\tau}-1 \leq e^{\tau}$. (15) holds for $F_{G}=1>0$. Therefore, in view of Theorem 3, (22) is generalized Hyers-Ulam-Rassias stable.

Here $\gamma=\frac{1}{3}$, by calculation, we have $M\left(\frac{1}{3}\right)=\frac{6}{5}, a_{\frac{1}{3}}=\frac{24}{25}$. Then $a_{\gamma} k_{f}=\frac{24}{25} \times 5=\frac{24}{5}>1$. Thus $a_{\alpha} k_{f}<1$ condition of Theorem 5 in [4] does not hold in this problem. Thus, ([4], Theorem 5) does not work even on $[0,2]$.

Author Contributions: M.F. and J.W. contributed to the supervision and project administration, K.L., M.F. and J.W. contributed to the conceptualization and methodology. All authors have read and approved the final manuscript.

Funding: This work is partially supported by the National Natural Science Foundation of China (11661016), Guizhou Provincial Science and Technology Foundation ([2020]1Y002), the Slovak Research and Development Agency under the Contract No.APVV-18-0308, and the Slovak Grant Agency VEGA No.1/0358/20 and No.2/0127/20.

Acknowledgments: The authors thank the referees for their careful reading of the article and insightful comments.

Conflicts of Interest: The authors declare no conflict of interest. 


\section{References}

1. Hyers, D.H. On the stability of the linear functional equation. Proc. Natl. Acad. Sci. USA 1941, 27, $222-224$. [CrossRef] [PubMed]

2. Rassias, T. On the stability of linear mappings in Banach spaces. Proc. Am. Math. Soc. 1978, 72, $297-300$. [CrossRef]

3. Jung, S.-M. A fixed point approach to the stability of differential equations $y^{\prime}=F(x, y)$. Bull. Malays. Math. Sci. Soc. 2010, 33, 47-56.

4. Liu, K.; Fečkan, M.; O’Regan, D.; Wang, J. Hyers-Ulam stability and existence of solutions for differential equations with Caputo-Fabrizio fractional derivative. Mathematics 2019, 7, 333. [CrossRef]

5. Başc1, Y.; Mısır, A.; Öğrekçi, S. On the stability problem of differential equations in the sense of Ulam. Results Math. 2020, 75, 6. [CrossRef]

6. Liu, K.; Wang, J.; Zhou, Y.; O'Regan, D. Hyers-Ulam stability and existence of solutions for fractional differential equations with Mittag-Leffler kernel. Chaos Solitons Fractals 2020, 132, 109534. [CrossRef]

7. Wang, J.; Lv, L.; Zhou, Y. Ulam stability and data dependence for fractional differential equations with caputo derivative. Elect. J. Qual. Theory Diff. Equ. 2011, 63, 1-10. [CrossRef]

8. Rezaei, H.; Jung, S.; Rassias, T. Laplace transform and Hyers-Ulam stability of linear differential equations. J. Math. Anal. Appl. 2013, 403, 244-251. [CrossRef]

9. Alqifiary, Q.; Jung, S. Laplace transform and generalized Hyers-Ulam stability of linear differential equations. Electron. J. Differ. Equat. 2014, 2014, 1-11.

10. Wang, J.; Li, X. A uniform method to Ulam-Hyers stability for some linear fractional equations. Mediterr. J. Math. 2016, 13, 625-635. [CrossRef]

11. da C. Sousa, J.; Capelas de Oliveira, E. Ulam-Hyers stability of a nonlinear fractional Volterra integro-differential equation. Appl. Math. Lett. 2018, 81, 50-56.

12. Wang, J.; Zhou, Y.; Fečkan, M. Nonlinear impulsive problems for fractional differential equations and Ulam stability. Comput. Math. Appl. 2012, 64, 3389-3405. [CrossRef]

13. Capelas de Oliveira, E.; Vanterler da C. Sousa, J. Ulam-Hyers-Rassias stability for a class of fractional integro-differential equations. Result Math. 2018, 73, 111. [CrossRef]

14. da C. Sousa, J.; Kucche, K.; Capelas de Oliveira, E. Stability of $\psi$-Hilfer impulsive fractional differential equations. Appl. Math. Lett. 2018, 88, 73-80.

15. da C. Sousa, J.; Capelas de Oliveira, E. On the Ulam-Hyers-Rassias stability for nonlinear fractional differential equations using the $\psi$-Hilfer operator. J. Fixed Point Theory Appl. 2018, 20, 5-21.

16. Shah, K.; Ali, A.; Bushnaq, S. Hyers-Ulam stability analysis to implicit Cauchy problem of fractional differential equations with impulsive conditions. Math. Meth. Appl. Sci. 2018, 41, 8329-8343. [CrossRef]

17. Ali, Z.; Zada, A.; Shah, K. Ulam stability to a toppled systems of nonlinear implicit fractional order boundary value problem. Bound. Value Prob. 2018, 2018, 175. [CrossRef]

18. Wang, J.; Zhang, Y. Ulam-Hyers-Mittag-Leffler stability of fractional-order delay differential equations. Optimization 2014, 63, 1181-1190. [CrossRef]

19. Liu, K.; Wang, J.; O'Regan, D. Ulam-Hyers-Mittag-Leffler stability for $\psi$-Hilfer fractional-order delay differential equations. Adv. Differ. Equal. 2019, 50, 1-12. [CrossRef]

20. Caputo, M.; Fabrizio, M. A new definition of fractional derivative without singular kernel. Prog. Fract. Differ. Appl. 2015, 1, 73-85.

21. Losada, J.; Nieto, J. Properties of a new fractional derivative without singular kernel. Prog. Fract. Differ. Appl. 2015, 1, 87-92.

22. Baleanu, D.; Mousalou, A.; Rezapour, S. On the existence of solutions for some infinite coefficient-symmetric Caputo-Fabrizio fractional integro-differential equations. Bound. Value Prob. 2017, 145, 1-9. [CrossRef]

23. Goufo, F.E.D. Application of the Caputo-Fabrizio fractional derivative without singular kernel to Korteweg-de Vries-Burgers equations. Math. Model. Anal. 2016, 21, 188-198. [CrossRef]

24. Atangana, A.; Nieto, J. Numerical solution for the model of RLC circuit via the fractional derivative without singular kernel. Adv. Mech. Eng. 2015, 7, 1-7. [CrossRef]

25. Moore, E.; Sirisubtawee, S.; Koonparasert, S. A Caputo-Fabrizio fractional differential equation model for HIV/AIDS with treatment compartment. Adv. Differ. Equat. 2019, 2019, 200. [CrossRef] 
26. Dokuyucu, M.; Celik, E.; Bulut, H.; Baskonus, H. Cancer treatment model with the Caputo-Fabrizio fractional derivative. Eur. Phys. J. Plus 2018, 133, 92. [CrossRef]

27. Başc1, Y.; Öğrekçi, S.; Mısır, A. On Hyers-Ulam stability for fractional differential equations including the new Caputo-Fabrizio fractional derivative. Mediterr. J. Math. 2019, 16, 131. [CrossRef]

28. Diaz, J.; Margolis, B. A fixed point theorem of the alternative, for contractions on a generalized complete metric space. Bull. Am. Math. Soc. 1968, 74, 305-309. [CrossRef]

(C) 2020 by the authors. Licensee MDPI, Basel, Switzerland. This article is an open access article distributed under the terms and conditions of the Creative Commons Attribution (CC BY) license (http://creativecommons.org/licenses/by/4.0/). 